\title{
SUMMARY OF 2012 ESC GUIDELINES
}

Marc J. CLAEYS ${ }^{1}$, MD; Patrick EVRARD ${ }^{2}$, MD, PhD; Olivier GURNÉ ${ }^{3}, \mathrm{MD}, \mathrm{PhD}$;

Georges MAIRESSE ${ }^{4}, \mathrm{MD}$; Yves VANDEKERKHOVE ${ }^{5}$, MD; Johan DE SUTTER ${ }^{6}, \mathrm{MD}, \mathrm{PhD}$;

Victor LEGRAND ${ }^{7}, \mathrm{MD}, \mathrm{PhD}$

${ }^{1}$ Antwerp University Hospital, Edegem, Belgium; ${ }^{2}$ UCL Mont-Godinne, Yvoir, Belgium; ${ }^{3}$ UCL Louvain, Brussels, Belgium;

${ }^{4}$ Cliniques du Sud Luxembourg, Arlon, Belgium; ${ }^{5}$ AZBrugge, Brugge; ${ }^{6}$ AZ Middelares, Gent, Belgium; ${ }^{7}$ ULG Liège, Belgium.

Keywords ESC-guidelines.

\section{INTRODUCTION}

During the ESC congress in August 2012 in Munich, the new ESC guidelines were presented and are now available on the ESC website (http://www.escardio.org/ guidelines).

The new guidelines cover management recommendations on the following items: acute myocardial infarction (particularly ST-elevation myocardial infarction), valvular heart disease, heart failure, atrial fibrillation and cardiovascular prevention.

The present document gives a summary of these guidelines and highlights the most important recommendations and changes in the management of these diseases.

It will help to increase awareness about the new guidelines and may stimulate to consult the full document for specific items. Ultimately, the authors hope that this document will enhance implementation of the new ESC guidelines in daily clinical practice.

Address for correspondence:

Prof. M. Claeys, MD, Dept. of Cardiology, Antwerp University Hospital, Wilrijkstraat 10, B-2650 Edegem, Belgium. E-mail: marc.claeys@ua.ac.be Received 18 October 2012; accepted for publication 23 October 2012.

\section{GUIDELINES FOR THE MANAGEMENT OF ACUTE MYOCARDIAL INFARCTION IN PATIENTS PRESENTING WITH ST-SEGMENT ELEVATION MYOCARDIAL INFARCTION (STEMI) \& THIRD UNIVERSAL DEFINITION OF MYOCARDIAL INFARCTION (MI) - EXPERT CONSENSUS DOCUMENT 2012}

\section{Summary by prof. dr. Patrick Evrard}

The new guidelines deal with the management of patients with STEMI and replace the document published in 2008. It covers different aspects such as emergency care and reperfusion therapy but also special subsets such as patient population, logistics, risk assessment and long-term therapies. After the 2000 and 2007 statements the third Global MI Task Force (Joint ESC/ACCF/AHA/WHF) now recognizes in the new definition of acute myocardial infarction (MI) that very small amounts of myocardial injury or necrosis can be detected by biochemical markers and/or imaging, particularly when such necrosis occurs in the setting of the critically ill, after percutaneous coronary procedures or after cardiac surgery. The most important messages can be summarized as follows:

\section{Criteria for acute myocardial infarction}

When there is evidence of myocardial necrosis in a clinical setting consistent with acute myocardial ischaemia any one of the following criteria meets the diagnosis for MI:

- Detection of a rise and/or fall of cardiac biomarker values [preferably cardiac troponin ( $\mathrm{cTn}$ )] with at least one value above the 99th percentile upper reference limit (URL) and with at least one of the following: 
- symptoms of ischaemia;

- new or presumed new significant ST-segment-T wave (ST-T) changes or new left bundle-branch block (LBBB);

- development of pathological Q waves in the ECG;

- imaging evidence of new loss of viable myocardium or new regional wall motion abnormality;

- identification of an intracoronary thrombus by angiography or autopsy.

- Cardiac death with symptoms suggestive of myocardial ischaemia and presumed new ischaemic ECG changes or new LBBB (cardiac biomarkers not available).

- Stent thrombosis associated with MI when detected by coronary angiography or autopsy in the setting of myocardial ischaemia and with a rise and/or fall of cardiac biomarker values with at least one value above URL.

- Revascularization procedure-related MI is arbitrarily defined by elevation of $\mathrm{cTn}$ values $(>5 \times \mathrm{URL})$ or a rise of cTn values $>20 \%$ (abnormal baseline) in case of PCI and by elevation of cTn values $(>10 \times \mathrm{URL})$ after CABG.

\section{Management of patients with STEMI}

The emergency care of patients presenting with STEMI starts at the point of first medical contact (FMC). Regional networks should be designed to deliver reperfusion therapy expeditiously and effectively, with efforts made to make primary PCI available within $60 \mathrm{~min}$ from the initial call to as many patients as possible. They must work to achieve and maintain the following quality targets:

- FMC to first ECG $\leq 10 \mathrm{~min}$; ECG monitoring thereafter.

- FMC to reperfusion therapy.

- For fibrinolysis $\leq 30 \mathrm{~min}$.

- For primary PCI $\leq 90 \mathrm{~min}$ ( $\leq 60 \mathrm{~min}$ if the patient presents within 120 minutes of symptom onset or directly to a PCI-capable hospital).

Reperfusion therapy is indicated in all patients with symptoms of $<12$ hours duration and persistent STsegment elevation or (presumed) new LBBB ( $>12$ hours if pain and ECG changes have been stuttering). Routine PCI of a totally occluded artery $>24 \mathrm{~h}$ after symptom onset in stable patients without signs of ischaemia (regardless of whether fibrinolysis was given or not) is not recommended.

Primary PCI with stenting is the recommended reperfusion therapy over fibrinolysis if it can be performed by an experienced team within 120 minutes of FMC. It is also indicated for patients with severe acute heart failure or cardiogenic shock. A dual antiplatelet therapy with aspirin and an ADP-receptor blocker is recommended with prasugrel, ticagrelor or clopidogrel (if the others are not available or contraindicated) and an injectable anticoagulant must be used - in order of preference: bivalirudin, enoxaparin or unfractionated heparin. GP IIb/IIIa inhibitors should be considered for bailout therapy if there is angiographic evidence of massive thrombus, slow or no-reflow or a thrombotic complication.

Fibrinolysis should be considered in patients presenting early ( $<2$ hours after symptom onset) with a large infarct and low bleeding risk, if time from FMC to balloon inflation is $>90 \mathrm{~min}$. The fibrinolysis should start in the pre-hospital setting with a fibrin-specific agent (tenecteplase, alteplase, reteplase). Dual antiplatelet therapy with aspirin and clopidogrel is indicated. The anticoagulation (enoxaparin, unfractionated heparin or fondaparinux in case of streptokinase) is recommended until revascularization or for the duration of hospital stay up to 8 days. After successful lysis the transfer to a PCIcapable centre is indicated in all patients 3-24 hours. Nevertheless, rescue $P C I$ is indicated immediately when fibrinolysis has failed $(<50 \%$ ST-segment resolution at $60 \mathrm{~min}$ ), emergency PCI in case of recurrent ischaemia or evidence of re-occlusion after initial successful fibrinolysis and emergency angiography with a view to revascularization is indicated in heart failure/shock patients after initial fibrinolysis. The use of fibrinolysis before planned primary PCI is not recommended.

There are special subsets. A high index of suspicion for MI must be maintained in women, diabetic and elderly patients with atypical symptoms. Both genders must be managed in similar fashion and special attention must be given to proper dosing of antithrombotics in elderly and renal failure patients. STEMI patients undergoing uncomplicated successful reperfusion therapy should be kept in capable coronary care unit (equipped to provide all aspects of care) for a minimum of 24 hours, after which they may be moved to a step-down monitored bed for another $24-48$ hours. The risk assessment of infarct size and resting LV function should be based on echocardiography. In case of multivessel disease or revascularization of other vessels stress testing or imaging for ischaemia and viability is indicated.

Lifelong control of risk factors, particularly smoking, must be stringent. As long-term treatment the antiplatelet therapy is indicated indefinitely and dual antiplatelet therapy up to 12 months. A fasting lipid profile must be obtained in all patients and a high-dose statin should be initiated or continued early after admission. Beta blockers, ACE inhibitors (ARB as an alternative) or aldosterone antagonists are indicated in patients with heart failure or left ventricular dysfunction. 


\section{GUIDELINES ON THE MANAGEMENT OF VALVULAR HEART DISEASE}

\section{Summary by prof. Marc Claeys}

This new document, which replaces the 2007 guidelines, is produced jointly by the ESC and the European Association for Cardio-Thoracic Surgery. It covers different aspects such as diagnosis, risk stratification and treatment and emphasizes the team approach (heart team consisting of cardiologist, cardiac surgeons, imaging specialists) in the decision-making process, particularly in high-risk patients. The most important management recommendations for patients with severe valvular heart disease can be summarized as follows:

1. Aortic regurgitation: surgery is indicated in symptomatic patients and in asymptomatic patients with evidence of cardiac repercussion (LVEF $<50 \%$ and/or LVEDD > $70 \mathrm{~mm}$ and/or LVESD > $50 \mathrm{~mm}$ ).

2. Aortic stenosis $\left(<\mathbf{1 . 0} \mathrm{cm}^{2}\right)$ : aortic valve replacement is indicated in symptomatic patients and in asymptomatic patients with depressed left ventricular function (EF $<50 \%)$ or abnormal exercise test (symptoms, blood pressure fall). AVR should be considered in asymptomatic patients with very severe stenotic and calcified valve or with rapidly progressive disease. The choice of AVR should be made by the heart team. Transcatheter aortic valve implantation (TAVI) is indicated in patients with a clear indication for AVR, but with a too high operative risk.

3. Mitral regurgitation: surgery (repair is preferred to replacement) is indicated in symptomatic patients with normal or moderately depressed left ventricular function $(\mathrm{EF}>30 \%)$ and in asymptomatic patients with evidence of cardiac repercussion (LVEF $<60 \%$ and/or LVESD $>45 \mathrm{~mm}$ ). Surgery should be considered in patients with a recent atrial fibrillation or with high pulmonary pressure at rest $(>50 \mathrm{mmHg}$ ) or during exercise $(>60 \mathrm{mmHg})$ particularly in patients with low operative risk and high likelihood of durable repair. In symptomatic patients with poor left ventricular function (EF $<30 \%$, mostly functional MR) surgery should be considered in case of low surgical risk and high likelihood of durable repair. The percutaneous mitral clip procedure may be considered in patients with symptomatic severe MR despite optimal medical therapy (including CRT if indicated), who fulfill the echo criteria of eligibility, are judged inoperable or at high surgical risk by the heart team.

4. Mitral stenosis $\left(<1.5 \mathrm{~cm}^{2}\right)$ : valve correction is indicated in symptomatic patients and should be considered in asymptomatic patients with high thromboembolic risk and/or abnormal exercise test. Percutaneous mitral commissurotomy is the preferred approach if valve anatomy is favourable.

5. Prosthetic valve thrombosis: in haemodynamic unstable patients with obstructive valve thrombosis, urgent surgery is indicated (option for fibrinolysis only if urgent surgery is not available). In haemodynamically stable patients optimalisation of antithrombotic treatment (heparin, aspirin) is first advocated. Only if therapy has failed should surgical intervention (or fibrinolysis in high surgical risk patients) be considered.

The final sections are dedicated to the choice of prosthetic valves and the management of VHD during noncardiac surgery and during pregnancy.

\section{GUIDELINES FOR THE DIAGNOSIS AND TREATMENT OF ACUTE AND CHRONIC HEART FAILURE}

\section{Summary by prof. Olivier Gurné}

This new document deals with the management of acute and chronic heart failure and replaces the "old" guidelines published in 2008. It covers different aspects from diagnosis to treatment. The most important changes, as compared to previous guidelines, can be summarized as follows:

\section{Diagnosis}

Distinction of heart failure with reduced ejection fraction (HF-REF) and with preserved ejection fraction (HF-PEF) has been clarified. For diagnosis, both require symptoms and signs of heart failure. HF-REF requires a reduced $\mathrm{EF}$ in addition. HF-PEF requires a normal or only mildly reduced EF but also a relevant structural heart disease (LV hypertrophy/LA enlargement) and/or diastolic dysfunction.

A new algorithm has been introduced for patients with suspected heart failure, with two alternatives, using echocardiography or natriuretic peptides in the first place, depending on their availability. New cut-off values have been introduced for BNP and NT-proBNP, depending on the presentation of the patient, based on their good negative predictive value. For an acute onset, values of less than $100 \mathrm{pg} / \mathrm{ml}$ for BNP and less than 300 for 
NT-proBNP make the diagnosis of heart failure unlikely. For non-acute onset, values of less than 35 and less than $125 \mathrm{pg} / \mathrm{ml}$ for BNP and NT-proBNP make the diagnosis of heart failure unlikely.

\section{Drug therapy}

a) HF-REF: indication for mineralocorticoid receptor antagonists (aldosterone, eplerenone) has been expanded, based on the EMPHASIS trial. They are now coming in second line, if the patient remains symptomatic despite ACE inhibitors and beta blockers, which remain the first-line treatment. Angiotensin II inhibitors are now more indicated as an alternative to ACE inhibitors if not tolerated. A new indication for the sinus node inhibitor ivabradine has been introduced based on the SHIFT trial, as a third line if the patient remains symptomatic, in sinus rhythm $\geq 70$ beats $/ \mathrm{min}$

a. HF-PEF: no treatment has yet been shown, convincingly, to reduce morbidity and mortality in these patients.

b) Devices: indication for CRT has been expanded for patients with mild symptoms (NYHA II), an EF $\leq 30 \%$ and a left bundle-branch block ( $\geq 130 \mathrm{msec}$ ). This treatment is considered as less certain in patients in atrial fibrillation or with a right bundle-branch block.

ICD is recommended in primary prevention in patients in class II-III with LV systolic dysfunction (EF $\leq 35 \%$ ) in ischaemic aetiology and $>40$ days after acute myocardial infarction (I A) and in non-ischaemic aetiology (I B).

A growing use of ventricular assist devices is also recognized, not only in bridge therapy but also in highly selected patients who have end-stage heart failure despite optimal treatment.

c) Surgery: based on the STICH trial, CABG is recommended in patients with systolic LV dysfunction for patients with angina and with not only significant left main stenosis, but also with 2- or 3-vessel coronary disease, including a left anterior descending stenosis. PCI may be considered as an alternative to CABG. CABG and PCI are not recommended in patients without angina and without viable myocardium. The role of isolated mitral valve surgery in patients with severe functional mitral regurgitation and severe LV systolic dysfunction who cannot be revascularized or have non-ischaemic cardiomyopathy, is questionable. In patients judged inoperable or at too high risk, percutaneous edge-to-edge repair may be considered.

d) Telemonitoring: the use of remote telemonitoring, using an implanted device or not, as well as serial monitoring of natriuretic peptides, is still uncertain for the follow-up of HF patients.

\section{GUIDELINES FOR THE MANAGEMENT OF ATRIAL FIBRILLATION}

\author{
Summary by dr. Georges H. Mairesse and dr. Yves \\ Vandekerkhove
}

At the time the 2010 ESC Guidelines for the Management of Atrial Fibrillation (AF) were issued, it was already known that an update would be necessary in 2012 to integrate the results of very important ongoing trials and European regulatory approvals of several new drugs. The most important evolutions can be summarized as follows:

1. Stroke and bleeding risk assessment: the $\mathrm{CHA}_{2} \mathrm{DS}_{2}-$ VASc score includes most common risk factors for stroke and should be used to determine the stroke risk in all patients with AF. However, instead of focusing on identifying the patients at high stroke risk, the actual management plan recommends to focus on identifying the patients at truly low risk (age $<65 \mathrm{y}$ and lone AF) who do not require any anticoagulation. Decision to anticoagulate should, however, be balanced against the bleeding risk and the HAS-BLED score has the best predictive value to identify the patients at high bleeding risk $(\geq 3)$.

2. Novel oral anticoagulants (NOACs): dabigatran, rivaroxaban and apixaban have all shown non-inferiority, and in some cases superiority, compared to warfarin to prevent thromboembolism. NOACs offer also better safety and convenience and should be considered instead of dose-adjusted vitamin K antagonists for most patients. The efficacy of aspirin is weak and its bleeding risk not significantly lower. Aspirin, in combination with clopidogrel, should be limited to the few patients who refuse any form of oral anticoagulation.

3. Left appendage closure: interventional percutaneous occlusion of the left atrial appendage has a role in patients with thromboembolic risk who cannot be managed in the long term using any form of oral anticoagulation.

4. Antiarrhythmic drug therapy: all antiarrhythmic drugs should only be used to relieve symptoms associated with AF. They should not be used for rate control only. Safety rather than efficacy should drive the choice of any antiarrhythmic drug.

Vernakalant is effective in cardioversion of patients with $\mathrm{AF} \leq 7$ days or $\leq 3$ days after cardiac surgery and provides rapid conversion to sinus rhythm in $50 \%$ of patients usually in 8-14 minutes. It should, however, be used with caution in fragile patients.

The use of dronedarone is limited to maintain sinus rhythm in patients with paroxysmal or persistent AF. It 
should not be given to patients with moderate or severe heart failure, and should be avoided in patients with less severe heart failure if an appropriate alternative exists.

5. Catheter ablation of atrial fibrillation: left atrial catheter ablation, targeting in the isolation of the pulmonary veins, is recommended as an alternative to antiarrhythmic drugs for patients with symptomatic recurrent paroxysmal AF on antiarrhythmic drugs, provided the procedure is performed by an experienced operator. In selected patients with no structural heart disease, ablation can even be reasonable as first-line therapy.

\section{GUIDELINES ON CARDIOVASCULAR DISEASE PREVENTION IN CLINICAL PRACTICE}

\section{Summary by prof. dr. Johan De Sutter}

This document is the result of the work of the $5^{\text {th }}$ Joint Task Force of the European Society of Cardiology and other societies on cardiovascular disease (CVD) prevention in clinical practice (chairman prof. J. Perk). It provides recommendations that are now shorter, better adapted to clinical needs and more practical as compared to previous versions of these guidelines. These recommendations are still highly relevant as atherosclerotic CVD, especially coronary heart disease, remains the leading cause of premature death worldwide and prevention has been shown to be responsible for over $50 \%$ of the reductions in observed CHD mortality.

The major new key message since the 2007 prevention guidelines can be summarized as follows.

1. Four levels of CVD risk are defined: very high, high, moderate and low risk

- Very high risk: subjects with any of the following:

a. Documented CVD by invasive or non-invasive testing

b. Diabetes mellitus (type 1 or type 2) with one or more $\mathrm{CV}$ risk factors and/or target organ damage (such as microalbuminuria) c. Severe chronic kidney disease (eGFR $<30 \mathrm{~mL}$ / $\mathrm{min} / 1.73 \mathrm{~m}^{2}$ )

d. A calculated SCORE $\geq 10 \%$ for 10 -year risk of fatal CVD

- High risk: subjects with any of the following

a. Markedly elevated single risk factors such as familial dyslipidaemia and severe hypertension

b. Diabetes mellitus (type 1 or type 2 ) but without $\mathrm{CV}$ risk factors or target organ damage

c. Moderate chronic kidney disease (eGFR 30-59 ml/ $\min / 1.73 \mathrm{~m}^{2}$ )

d. A calculated SCORE of $\geq 5 \%$ and $<10 \%$ for 10-year risk of fatal CVD

- Moderate risk: a calculated SCORE of $\geq 1$ and $<5 \%$ at 10 years

- Low risk: a calculated SCORE $<1 \%$ and free of qualifiers that would put them at moderate risk.

2. Risk factor screening should be considered in adult men $\geq 40$ years and in women $\geq 50$ years of age or if postmenopausal.

3. Multimodal intervention is recommended targeting several risk factors such as cholesterol, smoking (active and passive), physical exercise, blood pressure with the following specific targets:

- Blood pressure is $<140 / 90 \mathrm{mmHg}(<140 / 80 \mathrm{mmHg}$ for diabetic patients)

- Diabetes mellitus: HbA1c $<7 \%$

- Blood lipids : target LDL-cholesterol : $<1.8 \mathrm{mmol} / \mathrm{L}$ (70 mg/dl) for very-high-risk patients $<2.5 \mathrm{mmol} / \mathrm{L}(100 \mathrm{mg} / \mathrm{dl})$ for high-risk patients $<3.0 \mathrm{mmol} / \mathrm{L}(115 \mathrm{mg} / \mathrm{dl})$ for all others

4. Recommendations on programme provision after hospitalization

a. It is strongly recommended (class I, LOE B) that all patients with CVD must be discharged from hospital with clear guideline-oriented treatment recommendations to minimize adverse events.

b. It is strongly recommended (class IIa, LOE B) that all patients requiring hospitalization or invasive intervention after an acute ischaemic event should participate in a cardiac rehabilitation programme to improve prognosis by modifying lifestyle habits and increasing treatment adherence. 